\title{
Magic with rapamycin
}

Tuberous sclerosis complex (TSC) is caused by heterozygous mutations in the TSC1 or TSC2 genes and is characterized by a broad range of symptoms, such as mental retardation, seizures and behavioural problems. Ehninger et al. investigated hippocampus-dependent learning in mice with a heterozygous mutation in $T s c 2\left(T s c 2^{+/-}\right.$mice). They show that treatment with the mTOR inhibitor rapamycin rescues the learning abnormalities that are otherwise observed in these mice.

Individuals with a mild form of TSC appear healthy but have specific learning and memory problems. Previous work had revealed that $T s c 2^{+/-}$mice exhibit no spontaneous seizures or gross structural brain abnormalities, but their learning and memory had never been tested.

Initially the authors tested the hippocampus-dependent learning ability of $T s c 2^{+/-}$mice in three independent tasks. The mice showed significantly impaired spatial learning and working memory, and also showed no context-specific freezing responses in a context-conditioning paradigm, indicating that they had learning and memory impairments.

Next the authors used electrophysiological techniques to assess whether long-term potentiation (LTP) was affected in acute hippocampal slices taken from the mutant mice. They established that LTP could be induced at a much lower stimulation threshold in slices from $T s c 2^{+/-}$mice than in slices from wildtype mice. It is therefore conceivable that a lowered threshold for memory consolidation might lead to the learning impairments.

The authors then tested TSC 2 protein levels in the hippocampus, and found that they were reduced by $25 \%$ in the $T s c 2^{+/-}$mice. As TSC2 is a GTPase-activating protein that is known to indirectly inhibit the kinase mammalian target of rapamycin (mTOR), they investigated the phosphorylation levels of mTOR targets in the hippocampus. This revealed significantly higher levels of phosphorylation in the mutant mice, consistent with disinhibition of mTOR activity.

To determine whether the abnormal LTP or the learning impairments that were observed in the $T s c 2^{+/-}$mice were directly caused by increased mTOR activity, the authors intraperitoneally injected the mTOR inhibitor rapamycin before the training sessions for two of the learning tasks. Rapamycin reversed the deficits in the $T s c 2^{+/-}$mice in both tasks and also rescued the abnormal LTP when hippocampal slices were perfused with rapamycin before and during measurements. These results suggest that the learning impairments and the LTP phenotype of $T s c 2^{+/-}$mice

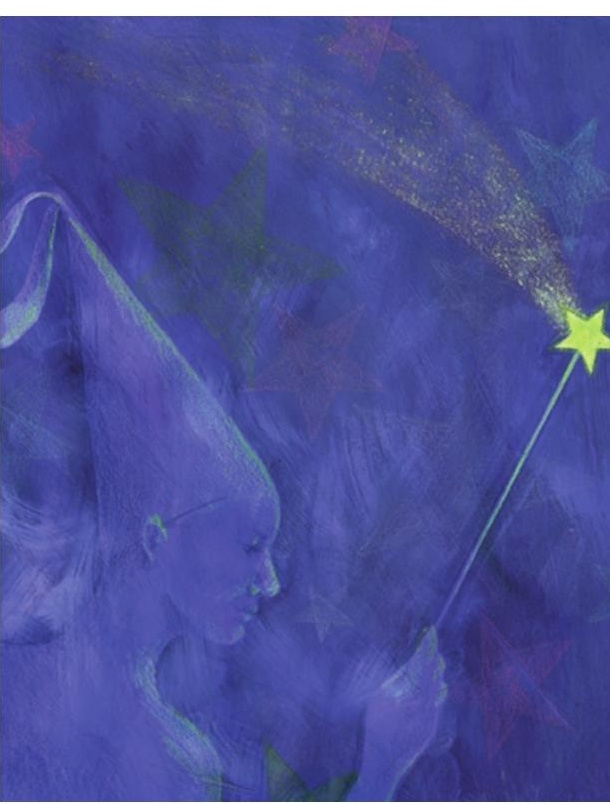

are directly caused by disinhibited mTOR signalling.

The authors showed not only that $T s c 2^{+/-}$mice are a valid model for the study of tuberous sclerosis, but also that rapamycin can reverse the deficits in LTP and learning in these mice. This suggests that the TSCmTOR signalling pathway underlies the learning deficits that are seen in the $\mathrm{Tsc2}^{+/-}$mice, and raises the possibility that mTOR inhibitors could be exploited as efficient treatment strategies for the cognitive defects associated with tuberous sclerosis.

Claudia Wiedemann

ORIGINAL RESEARCH PAPER Ehninger, D. et al. Reversal of learning deficits in a $\mathrm{TsC2}^{+ \text {+- }}$ mouse model of tuberous sclerosis. Nature Med. 22 Jun 2008 (doi: $10.1038 / \mathrm{nm} 1788$ ) 